\title{
Letter to the editor: does estrogen deprivation affect pelvic floor muscle contractility?
}

\author{
Chih-Ting Chang ${ }^{1} \cdot$ Chin-Ru Ker $^{1} \cdot$ Cheng-Yu Long $^{2}$ (D) \\ Received: 21 July 2019 / Accepted: 6 August 2019 /Published online: 23 August 2019 \\ (C) The International Urogynecological Association 2019
}

To the Editor,

We read with interest the recent article "Does estrogen deprivation affect pelvic floor muscle contractility?" by Hans Peter Dietz and co-authors [1]. This article concluded that estrogen deprivation is unlikely to be an independent predictor of pelvic floor muscle contractility. The severity of pelvic organ prolapse (POP) is a strong indicator of levator ani function and structural integrity, and in this article trans-perineal imaging diagnosed significant $\mathrm{POP}$ in $70.8 \%$ of all the attending population.

Advanced POP was clinically defined as POP-Q stage 2 or worse, with the most distal portion of the prolapse $>1 \mathrm{~cm}$ beyond the hymen [2].

The perineal ultrasound image of pelvic organs will be distorted in women with advanced POP. Because the bulging part of the POP will be pushed back into the vagina when applying a probe on the introitus, it may underestimate the POP staging. This might account for the higher incidence of POP through clinical examination $(76.4 \%)$ than ultrasound imaging (70.8\%). Although POP staging is not the main parameter of pelvic floor contractility in this article, it is still a useful predictor of levator ani function. We believe that clinical examination is a more appropriate tool for assessing POP staging than perineal ultrasound.
Since the serum level of estradiol will drop back to the baseline level hours or days after discontinuation of hormone therapy, a retrospective methodology may not be a good design for comparing postmenopausal women with prior hormone therapy [3]. The relationship between estrogen and pelvic muscle contractility is still being explored.

\section{References}

1. Dietz HP, Socha M, Atan IK, Subramaniam N. Does estrogen deprivation affect pelvic floor muscle contractility? Int Urogynecol J. 2019. https://doi.org/10.1007/s00192-019-03909-w

2. Persu C, Chapple CR, Cauni V, Gutue S, Geavlete P. Pelvic Organ Prolapse Quantification System (POP-Q) - a new era in pelvic prolapse staging. J Med Life. 2011;4(1):75-81.

3. Castelo-Branco C, Soveral I. Clinical efficacy of estradiol transdermal system in the treatment of hot flashes in postmenopausal women. Dove Press Journal. Research and Reports in Transdermal Drug Delivery $2014 ; 3: 1-8$.

Publisher's note Springer Nature remains neutral with regard to jurisdictional claims in published maps and institutional affiliations.
Cheng-Yu Long

urolong@yahoo.com.tw

1 Department of Obstetrics and Gynecology, Kaohsiung Medical University Hospital, Kaohsiung, Taiwan

2 Department of Obstetrics and Gynecology, Kaohsiung Municipal Siaogung Hospital, Kaohsiung, Taiwan 